\title{
Unzuverlässiges Erzählen und \\ Life-Writing. Zwei konfligierende \\ Konzepte in Vladimir Nabokovs \\ The Real Life of Sebastian Knight und \\ Thomas Manns Doktor Faustus
}

\section{Sonja Klimek}

Zusammenfassung Die beiden im Exil entstandenen Romane „The Real Life of Sebastian Knight" (1941) von Vladimir Nabokov und „Doktor Faustus“ (1947) von Thomas Mann sind zwei fiktionale Texte über das Schreiben nichtfiktionaler Künstler-Biographien. Da die fiktiven Biographen-Figuren selbst keine Künstler sind, wird in beiden Romanen der Blick von außen auf die künstlerische Kreativität dargestellt. Auf der Werkebene werden diese Versuche, einen anderen Menschen in seinem Gewordensein aus seiner Lebensgeschichte heraus zu verstehen, aber immer wieder mit den Mitteln der Groteske als unzulänglich bloßgestellt, nicht zuletzt durch die Lächerlichkeit ihrer den Prämissen und Strukturmerkmalen der anti-modernen Romanbiographien der 1920er und 1930er verpflichteten Erzähler-Figuren. Nabokovs besonders selbstreferentieller Roman ist ein Text über die Unmöglichkeit, einen anderen Menschen zu verstehen, indem man sein Leben erzählend rekonstruiert, was durch einen nicht offen unzuverlässigen, aber dennoch höchst zweifelhaften Ich-Erzähler narrativ gestaltet wird. Mann dagegen nutzt einen offen unzuverlässigen Erzähler, um einen Meinungsvorschlag zur Diskussion zu stellen, ohne dass Mann sich selbst als Autor zu dieser Position bekennen müsste. Beide Romane verfügen über die erzählerischen Traditionen der Romantik und bringen somit letztlich ihre Interpreten in die Position, in ihren Verstehensakten nun auch selbst Position zu beziehen, ob das Verstehen eines Künstlers - hier nicht seines Lebens, sondern eines seiner Werke - möglich sei.

\footnotetext{
S. Klimek $(\bowtie)$

Institut für Neuere Deutsche Literatur und Medien,

Christian-Albrechts-Universität zu Kiel,

Kiel, Deutschland

E-Mail: sklimek@ndl-medien.uni-kiel.de
} 
Die Frage nach der Zuverlässigkeit des Erzählens, wenn es darum geht, die Lebensgeschichte einer anderen Person zu schreiben, steht wie der Versuch jeden Fremdverstehens stets unter dem Verdacht, doch nur das Eigene im Fremden wiederzuerkennen. Handelt es sich bei dem Porträtierten auch noch um einen Künstler, so tritt zusätzlich die Frage nach der Verstehbarkeit von Kunst ganz generell hinzu. Handelt es sich drittens nicht etwa um ein Sachbuch, d. h. um eine nicht-fiktionale Biographie über einen realen Künstler, sondern um einen Künstlerroman oder eine Künstlernovelle über eine fiktive Künstlerfigur, so enthält der Text obendrein auch noch eine selbstbezügliche Komponente.

Bei den Romanen Doktor Faustus von Thomas Mann und The Real Life of Sebastian Knight von Vladimir Nabokov handelt es sich um solche fiktionalen Texte, die ein empirischer Autor (als realer Künstler) über eine fiktive Figur geschrieben hat, welche wiederum die - in ihrer Welt nicht-fiktionale - Lebensbeschreibung einer Künstler-Figur verfasst. Es handelt sich mithin um Texte über das Schreiben, genauer gesagt: um fiktionale Texte über das (an sich nichtfiktionale) ,Life-Writing'. In beiden Romanen rekonstruiert ein Vertrauter eines verstorbenen Künstlers postum dessen Lebensgeschichte. In beiden Büchern ist dieser Erzähler nicht etwa eine ,verwandte Seele', d. h. ebenfalls ein Künstler, sondern in beiden Fällen sind die Biographen gänzlich unkünstlerische Figuren, die erst durch ihren Akt des Biographierens überhaupt in den Bereich der Kreativität eintreten. Insofern spielt in beiden Werken die Faszination für den Künstler als den Anderen, für die Kunst als das dem eigenen Wesen unheimlich-faszinierend Fremde eine zentrale Rolle: Was ist Kreativität?, fragen sich die bürgerlichen Autoren der Lebensbeschreibungen dieser Künstler. Wo kommt sie her? Kann man als ,Normalsterblicher' ein ,Genie“ überhaupt verstehen?

Die Zuverlässigkeit des eigenen Erzählens in Bezug auf die Lebensgeschichte des praktischerweise bereits verstorbenen und insofern für Selbsterklärungen nicht mehr verfügbaren Künstlers infrage zu stellen, gehört von vorne herein zu solchen erzählerischen Konstruktionen, denn die von den Erzählerfiguren zum Ausgangspunkt gemachte Genieästhetik impliziert als grundlegende Grenze aller Verstehensbemühungen ja gerade die essentielle Andersartigkeit des Beschriebenen, die Überzeugung, dass den Künstler ohnehin kein ,praktisch Tätiger‘ je ganz verstehen könne. An diesem Punkt jedoch spielen beide Erzählerfiguren ihre eigene Biographie aus: Aufgrund ihrer persönlichen, bis in die Kindheit hineinreichenden besonderen Beziehung zu den einsamen Genies können eben nur sie selbst - wenn überhaupt irgendjemand - ein realistisches Bild des Verstorbenen zeichnen. Nur sie verfügen über das dafür nötige privilegierte biographische Wissen.

In den beiden im Folgenden behandelten Romanen spielt neben der Biographie der Künstler- und der partiellen Autobiographie der Erzählerfigur aber noch eine dritte Lebensgeschichte zentral mit hinein, nämlich die des jeweiligen empirischen Autors. 


\title{
1 Die gefährliche Suche nach des Rätsels Lösung. Nabokovs The Real Life of Sebastian Knight
}

\begin{abstract}
Sebastian Knight was born on the thirty-first of December, 1899, in the former capital of my country. An old Russian lady [...] happened to show me in Paris the diary she had kept from the past. [...] Therefore I am able to state that the morning of Sebastian's birth was a fine windless one, with twelve degrees (Reaumur) below zero... this is all, however, that the good lady found worth setting down. (Nabokov 1959 [1941], S. 5)
\end{abstract}

Mit dieser Rede des figürlich ausgestalteten, homodiegetischen Erzählers, der sich selbst bloß beiläufig „V.“ nennt, beginnt Nabokovs Roman The Real Life of Sebastian Knight. Der erste Satz enthält die klassische Eröffnung einer Biographie, die das Leben einer anderen Person üblicherweise, von der Wiege bis zur Bahre' erzählt. Doch bereits der zweite Satz lässt sich als metanarrativer Kommentar lesen: Der Ich-Erzähler, von dem die Leser später erfahren, dass er selbst der jüngere Halbbruder des Biographierten sei, legt Rechenschaft darüber ab, wieso er eigentlich von Umständen erzählen könne, deren Zeuge er nicht selbst gewesen sein kann, da sie entweder - wie im Fall des Wetters am letzten Tag des Jahres 1899 - vor seiner eigenen Geburt stattgefunden haben oder weil sie - wie später im Roman noch häufig - in seiner Abwesenheit passiert sind.

Es gehört nicht viel dazu, herauszufinden, dass auch der empirische Autor, Vladimir Nabokov, wie der titelgebende Protagonist des Romans 1899 in einer ,früheren Landeshauptstadt' geboren wurde: Die deutschsprachige Taschenbuchausgabe etwa enthält auf dem Frontispiz, gegenüber dem Titelblatt, eine kurze Autorenvita als Peritext, der mit dem Satz beginnt: „Vladimir Nabokov wird am 23. April 1899 in St. Petersburg geboren." (Nabokov 1999, S. 2) Die Nichtkünstler-Figur „V.“" schreibt also einen Roman über eine Schriftsteller-Figur, der der empirische Autor erkennbare Versatzstücke aus seiner eigenen Biographie verliehen hat. ${ }^{1}$

Es handelt sich bei The Real Life of Sebastian Knight um Nabokovs ersten auf Englisch verfassten Roman. Alle vorherigen literarischen Texte hatte der ExilRusse noch in seiner Muttersprache verfasst und in russischen Exil-Verlagen für ein russischsprachiges, ebenfalls emigriertes Zielpublikum veröffentlicht. Die Niederschrift erfolgte um den Jahreswechsel 1938/1939 in Paris, mithin in jener Zeit kurz nach der Reichspogromnacht vom 9. auf den 10. November 1938, als der mit einer russischen Jüdin verheiratete Nabokov sich dringendst um ein Auskommen als Schriftsteller oder wenigstens um eine kleine Anstellung an irgendeinem College oder Museum in Übersee bemühte - möglichst weit weg sowohl vom Deutschen Reich als auch von der Sowjetunion. Mit dem Sprachwechsel von Russisch zu Englisch zielte Nabokov erstmals auf eine völlig neue Lesergruppe.

\footnotetext{
${ }^{1}$ Dass Nabokov seinem Sebastian Knight nicht einfach an seinem eigenen Geburtstag, sondern tatsächlich am letzten Tag des scheidenden Jahrhunderts zur Welt kommen lässt, lädt zu der Interpretation ein, hier solle durch Zuspitzung eine Figur als letzter Vertreter, als Überbleibsel einer unwiederbringlich vergangenen Ära inszeniert werden.
} 
The Real Life of Sebastian Knight erschien schließlich 1941 in den USA und wurde dort 1959 noch einmal in überarbeiteter Neufassung herausgegeben.

Der Roman handelt von ,V.“s teilweise detektivischer Suche nach Quellen und Zeugen, denn eigentlich standen sich die Halbbrüder, wie die Leser erfahren, gar nicht nahe. Zu Beginn der Handlung weiß „V.“ fast nichts über das Leben Sebastians. Für eine kohärent erzählbare Lebensgeschichte oder gar eine WerkBiographie reichen seine Kenntnisse jedenfalls nicht. Eine Biographie des Verstorbenen existiert dagegen durchaus schon, und zwar in Form eines erst kürzlich erschienenen Werks aus der Feder von Sebastians ehemaligem Sekretär namens Goodman. „V.“ inszeniert sein eigenes Buch als Gegenentwurf zu dem Goodmans, der Sebastian Knights künstlerisches Schaffen und Scheitern vor dem zeithistorischen Hintergrund der Oktoberrevolution und dem politischen wie geistigen Klima in den Jahren danach deutet und damit - so „V.“ - dessen künstlerischen Wert völlig verfehle.

Warum sollten die fiktionsinternen Leser dem Erzähler „V.“ aber mehr Glauben schenken als der Biographie dieses Goodman (die die realen Leser von Nabokovs Roman zudem ja gar nicht kennen können)? Es erscheint von Anfang an zweifelhaft, warum gerade ,V.“ für das Schreiben dieser Biographie der bessere Mann sein sollte - hat er doch als Angestellter im mittleren Management einer französischen Firma keinerlei Erfahrung mit dem Schreiben. Freimütig gibt „V.“ denn auch zu, extra einen Kurs in Creative Writing absolviert zu haben, ehe er sich an diese Aufgabe herangewagt habe (vgl. Nabokov 1959 [1941], S. 34 f.). Was „V.“ antreibt, ist offenbar eine diffuse Neid- und Hassliebe-Beziehung zu dem verstorbenen Halbbruder. Dazu kommt „V.“s Wut über den Ansatz Goodmans, der Sebastian Knight - ,V.“s Meinung nach - völlig verkenne, da ihm eben fehle, was „V.“ zu besitzen behauptet: privilegiertes biographisches Wissen aus der gemeinsam verbrachten Kindheit sowie die darauf aufbauende Fähigkeit, Knights fiktionale Werke auf richtige Weise autobiographisch zu interpretieren.

I have not been able to obtain a picture of the house where Sebastian was born, but I know it well, for I was born there myself, some six years later. We had the same father: he had married again, soon after divorcing Sebastian's mother. Oddly enough, this second marriage is not mentioned et all in Mr. Goodman's Tragedy of Sebastian Knight [...]; so that to readers of Goodman's book I am bound to appear non-existent - a bogus relative, a garrulous impostor; but Sebastian himself in his most autobiographical work (Lost Property) has some kind words to say about my mother [...]. (Nabokov 1959 [1941], S. 6)

Der Ich-Erzähler behauptet qua exklusivem Geheimwissen, in den Werken Sebastian Knights viele autobiographische Stellen erkennen zu können. Ständig zitiert er in seiner Biographie aus den (wohlgemerkt fiktionalen) Werken des Portraitierten, um dessen Leben zu beschreiben. Dabei ist sein eigener privilegierter Status durch „V.“s Nicht-Vorkommen in Goodmans Biographie zweifelhaft. Man könnte dem fiktionsinternen Ich-Erzähler mit seiner biographischen Methode des Widererkennens verborgener autobiographischer Versatzstücke in Knights Werken misstrauen - wenn man nicht gleichzeitig als Roman-Leser bemerken würde, dass auch das Werk Nabokovs solche mal mehr, mal weniger gut kaschierten autobiographischen Versatzstücke enthält, die von 
„V.“ seinem Bruder Sebastian unterstellte Methode also tatsächlich auch vom empirischen Autor praktiziert wird:

Nachdem Nabokov, Sohn eines reichen Petersburger Journalisten und DumaAbgeordneten, 1917 knapp 18-jährig mit seinen Eltern und Geschwistern wie „V.“ und Sebastian mit ihrer Mutter - aus Russland geflohen war, hatte er zunächst - wie Sebastian - in Cambridge russische und französische Sprache und Literatur studiert, ehe er sich in Berlin, der damals größten ExilrussenCommunity, niederließ. Dort versuchte Nabokov, sich als russischsprachiger Autor zu etablieren. Es gab im Berlin der 1920er Jahre russische Verlage, Zeitungen, Theater und eine rege an diesem kulturellen Leben teilnehmende Leserschaft. Diese Jahre in Deutschland, 1922 bis 1937, gehören zu den geheimnisvollsten im Leben Nabokovs. Angeblich habe er, das Sprachgenie, in dieser ganzen Zeit kein Deutsch gelernt, kaum Kontakt zu Deutschen gehabt und sich auch sonst nicht weiter mit Politik beschäftigt (vgl. Zimmer 1999). Er, dessen ganzes Leben durch die äußeren politischen Umstände immer wieder durcheinandergewirbelt wurde, habe - so lange Zeit die einhellige Forschungsmeinung - diesem äußeren Wirrsal durch einen hochmütigen Rückzug in den Ästhetizismus, in eine absolute Kunst getrotzt. 1925 heiratete Nabokov in Berlin die russische Jüdin Vera Slonim, 1934 kam der gemeinsame Sohn Dmitrij zur Welt. Warum die Familie nach 1933 in Berlin blieb, erscheint in der Rückschau rätselhaft. Die Repressionen nahmen merklich zu. Unter anderem verlor Vera aus rassenidiologischen Gründen ihre Anstellung. Lange Zeit vermutete die Nabokov-Forschung, der weltfremde Autor habe einfach nicht mitbekommen, was sich um ihn herum abspielte. Dazu passt dann eine auch heute noch dominante Lesart seiner Werke als hochartistische, selbstreferentielle und metafiktionale Vexierspiele, die die Literatur mit sich selbst in ihrem eigenen Kosmos treibe.

Diese These vom weltfremden Sprachgenie ist aber mittlerweile aufgrund der Quellenlage widerlegt: Nabokov, der seit seiner Flucht aus Russland versucht hatte, sich eine Existenz als russischsprachiger Exil-Schriftsteller aufzubauen und wie viele Exil-Russen der Zwischenkriegszeit nur auf den Zusammenbruch der Sowjetunion gewartet zu haben scheint (vgl. Boyd 1990, S. 495), musste bereits in den ersten Jahren der Nazizeit erkennen, dass seine Familie sich in Berlin erneut in einer lebensbedrohlichen Lage befand, aus der er sie nur befreien konnte, indem er für sich Verdienstmöglichkeiten im ferneren Ausland auftat. Versuche, in England, Frankreich oder auch den USA ein Auskommen und somit eine Aufenthaltsbewilligung zu erlangen, lassen sich inzwischen seit den frühen 1930er Jahren nachweisen und werden bis 1937 zahlreicher und dringlicher. Als sich abzeichnete, dass Nabokov auf diesem Weg keine Einreisegenehmigung für seine Familie und sich in den USA erwirken konnte, schrieb er unter Zeitdruck seinen ersten englischen Roman, The Real Life of Sebastian Knight, den er für einen Wettbewerb in Großbritannien einreichen wollte (vgl. Boyd 1990, S. 496). Um sich mit seinem Englisch jedoch keine Blöße zu geben, arbeitete Nabokov den Roman nach der ersten Niederschrift dann doch noch einmal minutiös mit einer muttersprachigen Bekannten durch, was die Frist für den englischen Schreibwettbewerb jedoch überschritt (vgl. Boyd 1990, S. 503). 
1937 gelang es Nabokov endlich während einer Lesereise, Frau und Kind über Ungarn aus dem Deutschen Reich herauszuholen. Es folgte ein zweijähriges Wanderleben in zahlreichen französischen Pensionen und in prekären materiellen Verhältnissen. Im Mai 1940 bekamen die Nabokovs endlich ein Visum und konnten zu dritt von Saint-Nazaire nach New York übersetzen, und zwar buchstäblich auf dem ,letzten Dampfer“, auf dem Emigranten Frankreich noch rechtzeitig vor dem Einmarsch der deutschen Truppen verlassen konnten (vgl. Zimmer 1999 [1960], S. 271).

Unter derart dramatischen Lebensbedingungen verfasst, überarbeitet und angelsächsischen Verlagen angeboten, beschäftigt sich The Real Life of Sebastian Knight erneut mit Nabokovs Kern-Themen, um die viele seiner früheren wie späteren, fiktionalen wie autobiographischen Texte kreisen: die Frage, wie sich Identität auch unter den gegenwärtigen Erfahrungen der Moderne, des Vertriebenseins und der Emigration narrativ verbürgen oder erzeugen lasse - und wie wie dann in ihrer ganzen Konstruiertheit doch wieder in sich zusammensacke. Auch Sebastian Knight ist trotz seines Namens, den er erst nachträglich von seiner englischen Mutter angenommen hat, in Russland geboren und aufgewachsen, ein Exilant wie Nabokov, obwohl zumindest für Knights Biographen „V.“ und somit auch für die Leser des Romans nie so ganz klar wird, welche Rolle diese doppelte Herkunft - einerseits Engländer über die Mutter und aus Wahl, andererseits Russe über den Vater und durch die in Russland verbrachte Kindheit - für Sebastian eigentlich gespielt haben mag. Auch dieses Motiv kann zumindest teilweise als Versatzstück aus Nabokovs eigenem Leben verstanden werden. Im Roman kommen zwei Probleme zusammen: einerseits die Frage nach der Erzählbarkeit eines Lebens bzw. nach der reflektierten narrativen Konstruierbarkeit, Rekonstruierbarkeit, Versicherbarkeit und Unmöglichkeit von stabiler Identität unter den Bedingungen der Moderne allgemein und unter denen von Flucht und Exil ganz konkret. Andererseits die Frage nach der Verstehbarkeit eines Künstlers und seiner Werke.

Romanbiographien, d.h. offen fiktionale Biographien über fiktive Personen ebenso wie Sachbuch-Biographien in romanhaftem Stil über reale Personen, gehörten zu den kommerziell erfolgreichsten Gattungen der Zwischenkriegszeit. Stefan Zweig etwa wurde in den 1920er und frühen 1930er Jahren mit Werken wie Marie Antoinette, Erasmus von Rotterdam und Mary Stuart weltberühmt. Dieser Erfolg machte ihm die relativ rasche Aufnahme in den USA möglich wenn auch nicht den dauerhaften Verbleib. Nach dem eher traditionellen Erzählprinzip „Wie wurde jemand zu dem, als den man ihn kennt?“ setzten solche Erzählwerke ein relativ stabiles Konzept von Charakter voraus, dem der Biograph durch alle Zufälle des Lebens hindurch bei seiner Formung zuschaute; nichts wurde dazuerfunden, „,nur das Vorhandene“ , ausgedeutet“ (Zweig 2011, S. 186). Spätestens seitdem der Einfluss von Freud auf das Konzept des Selbst stärker wurde, kehrten sich moderne Autoren zunehmend vehement von dieser Art, Biographien zu schreiben, ab. Virginia Woolf etwa propagierte, in der Nachfolge James Joyces und in der Tradition selbstreflexiven Erzählens seit Laurence Sterne, die sogenannte „New Biography“, die der Fragmentarisierung von Erfahrung in 
der entfremdenden Moderne durch eine Fragmentarisierung der Schreibweise zu entsprechen suchte. Während bisher davon ausgegangen wurde, dass das Leben eines Menschen, sein Wesen, aus Dokumenten durch minutiöse Recherche rekonstruierbar sei, betonte Woolf stark die Grenzen der Biographie: Das Wesentliche, das Innere eines Autors, seine Gedanken während seiner Lektüreerfahrungen, seine Gründe und der ganze Komplex seines Unbewussten, seien dem Biographen ohnehin nie zugänglich (vgl. Lee 1977/2010). Erinnerungen können täuschen. Dokumente können falsche Datenlagen vorspiegeln und Kontexte aufscheinen lassen, die für die Entstehung des Werkes selbst oder für dessen angemessenes Verständnis irrelevant sind. Das ist längst ,status quo “ der modernen Autoren. ${ }^{2}$ Nabokov knüpft bei diesen Themen der Klassischen Moderne an, denn im Roman The Real Life of Sebastian Knight scheitern „V."s biographisch ,ausdeutende" Annäherungen an Leben und Werke seines verstorbenen Halbbruders immer wieder auf groteske Weise: Wo immer „V.“ sich seinem Bruder nahe fühlt, ist er in Wirklichkeit bloß ganz tief in seine eigenen Vorstellungen verstrickt - so etwa in der Szene, als „V.“ mit letzter Kraft ans Sterbebett Sebastians eilt und die Gegenwart des durch einen Wandschirm seiner Sicht Entzogenen genießt: „That gentle breathing was telling me more of Sebastian than I had ever known before." (Nabokov 1959 [1941], S. 203) - Der wohlige Zustand von Verbundenheit und Verstehen wird auf drastische Weise mit der Realität konfrontiert, als ,V.“ von der beschämten Krankenschwester erfährt, dass es sich um eine Verwechslung gehandelt habe, der Mann im Bett heiße „Kegan“, und Sebastian Knight sei bereits am Vortag verstorben (vgl. Nabokov 1959 [1941], S. 204).

Diese Situation spiegelt wiederum eine frühere Szene, allerdings nicht aus „V.“s, sondern aus Sebastians Leben, oder besser: aus seinem Werk „Lost Property“, aus dem „V.“ eine längere Passage zitiert und als autobiographischen Bericht deutet: Demnach hatte Sebastian Knight (oder der Ich-Erzähler aus „Lost Property“) als junger Mann einmal eine gewisse Pension im südfranzösischen Roquebrun aufgesucht, in der seine Mutter angeblich verstorben war, um sich ihr dort noch einmal ganz nahe zu fühlen. Auch bei Sebastian war dieser Plan zunächst scheinbar aufgegangen. Beim Anblick eines Blumenbeetes im Garten der Pension sah der junge Mann quasi ihren Geist im Garten umherwandeln: „Gradually I worked myself into such a state that for a moment the pink and green seemed to shimmer and float as if seen through a veil of mist. My mother, a dim slight figure in a large hat, went slowly up the steps which seemed to dissolve into water.“ (Nabokov 1959 [1941], S. 19 f.) In Sebastians Buch erfährt der IchErzähler (der vielleicht mit Sebastian identisch, vielleicht aber doch nur eine fiktive Figur ist) dann jedoch später, dass er sich in einem gleichnamigen Ort im falschen Departement aufgehalten habe, mithin die gesamte Vision nur Produkt

\footnotetext{
${ }^{2}$ Vgl. hierzu etwa den Beitrag von Hans-Harald Müller über die Romane Leo Perutz' im vorliegenden Band.
} 
seiner Phantasie, keineswegs einer mit dem Ort verbundenen magischen Präsenz der Verstorbenen gewesen sei (vgl. Nabokov 1959 [1941]).

Nabokovs Roman ist ein Roman über die Unmöglichkeit, einen anderen Menschen $\mathrm{zu}$ verstehen, indem man sein Leben rekonstruiert (Quellen auswertet, an die Orte seines Lebens und Sterbens reist, seinem vermeintlichen Atem lauscht, seine Werke biographisch liest etc.) und über das dringende menschliche Bedürfnis, es dennoch zu versuchen. Dabei werden zwei unterschiedliche interpretationstheoretische Ansätze gegeneinander ausgespielt: Der von „V.“ abgelehnte ,Schreiberling' Goodman steht für eine sozialgeschichtliche Kontextualisierung der Werke und ihres Verfassers. „V.“ dagegen ist zwar bloß ,angelernter“ Literat, betont aber umso dringlicher den Ästhetizismus, die Zeitenthobenheit und geniehafte Einmaligkeit der Kunstwerke Sebastian Knights, die letztlich immer nur um ihren Autor gekreist hätten. „V.“ benutzt daher die literarischen Texte als Steinbrüche, um das ihm völlig verschlossene Innenleben seines Bruders durch Einfühlung verstehen zu können. Dabei liest er alle fiktionalen Erzähltexte seines Halbbruders als Enigmen, in denen er die ,romantische Urschrift" wie im ,Buch der Natur' zu lesen sucht und darin die Antwort auf die letzten Fragen zu finden hofft: „The answer to all questions of life and death, 'the absolute solution' [...], the intricate pattern of human life turns out to be monogrammatic, now quite clear to the inner eye disentangling the interwoven letters." (Nabokov 1959 [1941], S. 178 f.) Dieses romantische Konzept einer ,absolute solution“ (einer ,absoluten Lösung'), eines großen, verborgenen Total-Sinns, den der Mensch unablässig zu finden und aus den als ,Zeichen“ interpretierten Details des Lebens zu entziffern bemüht sei - und von dem der moderne Mensch überzeugt ist, dass es ihn gar nicht gibt und von dem der Ästhetizist glaubt, dass nur die Kunst als modernes Substitut der Religion ihn suchend erschaffen könne (vgl. King 2012, S. 58-60) -, ist laut Caulton (2013) das eigentliche Thema des Romans The Real Life of Sebastian Knight. Der Roman handelt zwar von „V.s“ von ihm selbst erzählter Suche nach dem ,wahren Leben“ des Sebastian Knight. Der Roman bedeutet jedoch, so Caulton, dass die Suche nach dem ,wahren Leben“ von wem auch immer nicht nur vergeblich ist - das wäre ja nichts Neues nach ein paar Jahrzehnten modernen Erzählens und „New Biography“ -, sondern dass diese Suche, oder vielmehr das dahinterstehende Konzept eines verborgenen Sinns hinter allen zufälligen ,Zeichen“, kein harmloses ,Glasperlenspiel' ist, das die Kunst mit sich selbst spielt, sondern bereits gefährlich: Eine solche Sehnsucht mag menschlich erscheinen, sie ist jedoch stets Ausdruck einer totalitären Weltsicht. „I sometimes feel when I turn the pages of Sebastian's masterpiece“, so schreibt „V.“ über Sebastians letzten Roman, The Doubtful Asphodel, ,that the 'absolute solution' is there, somewhere, concealed in some passage I have read too hastily, or that it is intertwined with other words whose familiar guise deceived me." (Nabokov 1959 [1941], S. 180) „V.“ lernt am Ende seines Romans, dass es in Wirklichkeit dieses ,Gefühl' selbst ist, dass ihn getrogen hat - eine Lektion, die der Ich-Erzähler von Knights Roman „Lost Property“ ja ebenfalls zu lernen hatte, als dieser das Leben seiner jung verstorbenen Mutter zu rekonstruieren und sich ihr nahe zu fühlen versucht hatte. „V.“, der in seiner Unfähigkeit und Schreibbeflissen- 
heit eine komische Figur darstellt, ist zwar als fiktionsinterner Erzähler erst einmal der erste sich anbietende Kommunikationspartner der Leser von Nabokovs The Real Life of Sebastian Knight. Für die Leser wirkt „V.“ aber zunehmend als eine unglaubwürdige Figur, deren biographischer Geniekult mit der einfühlenden Methode letztlich immer nur neue ,Fiktionen“ des Verstehens herbeiführt. Am Ende kommt „V.“ seinem verstorbenen Halbbruder nicht nur mehr nahe, sondern wähnt sich gar identisch mit ihm: „The end, the end. [...] but the hero remains, for, try as I may, I cannot get out of my part: Sebastian's mask clings to my face, the likeness will not be washed off. I am Sebastian, or Sebastian is I, or perhaps we both are someone whom neither of us knows." (Nabokov 1959 [1941], S. 205) Ist „V.“ also Vladimir Nabokov? Oder ist Sebastian Knight das Alter Ego Nabokovs? Oder sind beide, so das häufig als metafiktionaler Illusionsbruch gedeutete Ende des Romans, letztlich ein und derselbe, nämlich als literarische Figuren Teil des Geistes ihres Autors und somit jeweils bloß andere Abspaltungen von Nabokovs komplexer Persönlichkeit? - Wie so oft in seinen Werken, so hat Nabokov auch hier jede mögliche Deutung sogleich wieder durch eine weitere ,turn of the screw' kunstvoll aufgehoben. Die Jagd nach dem Absoluten, dem verborgenen Sinn endet letztlich, das wussten schon die Autoren der Frühromantik, in Identitätsverlust, Metafiktion, in unendlicher Selbstbespiegelung, infinitem Regress oder, wie Novalis es in seinen Fragmenten formulierte, in ,qualitativer Potenzierung“ (vgl. Fricke 2002, S. 144). Das große Geheimnis hinter der ,Schrift der Natur", die Lösung für das Schachproblem des schwarzen Springers (auf Englisch heißt der Springer „Knight“), die Erklärung für „V.“s obskures Verhältnis zu Sebastian - all dies findet sich im Romantext nicht.

V. parodies the assumption that there can be an open sesame to another's past, and shows
us the barred doors and the trompe-l'oeil archways that have thwarted him. [...] V.‘s
self-conscious parody of the limitations of biography turns his own work into a playful
meditation on the inviolability of the individual and the mystery of the past. (Boyd 1990,
S. 500)

Nabokovs Roman nimmt all diese romantisch inspirierten Lektüre-Erwartungshaltungen in seinen Roman auf - und lässt sie dann doch ins Leere laufen. Auch die Kunst schafft hier nicht den Sinn, den das Leben an sich nicht bieten kann. In den Roman eingestreut finden sich nämlich mehrere Stellen, an denen man merkt, dass sich Sebastians Leben mitnichten in einem ahistorisch-sterilen Ego-Kosmos abspielte, sondern dass es eben doch - wie Goodman, „V."s HassGegner, behauptete - bestimmt wurde (und dass übrigens auch „V.“"s eigenes Leben bestimmt wird) durch die Konstellationen von Wirtschaft, Politik und dem Meinungsklima um ihn herum: Da wäre zunächst jene kleine Notiz in der Telefonkabine eines Pariser Cafés, in dem „V.“ auf der verzweifelten Reise ans Sterbebett seines Halbbruders strandet. Die Gedanken an den Gesuchten werden erzählerisch ohne Überleitung kontrastiert mit der Irritation über das politische Klima, in dem diese Suche stattfindet: „Would I never get to Sebastian? Who were those idle idiots who wrote on the wall 'Death to the Jews' or 'Vive le front populaire', or left obscene drawings?“ (Nabokov 1959 [1941], S. 197). 
Das Ankommen bei Sebastian, in seinem ,Real Life“ in Gestalt seines ruhigen Atems hinter dem Vorhang, steht hier für die ,,absolute solution“, die Entzifferung jener ,Urschrift" der Natur oder jenes Rätsels des Lebens, das die Werke des Halbbruders ,V.““ aufzugeben scheinen. Doch die Reise wird retardiert gerade in Paris, dem Ort, an dem die Familie des empirischen Autors Vladimir Nabokov zur Zeit der Roman-Niederschrift unter dem grassierenden Antisemitismus zu leiden hatte und der sie in Anbetracht des drohenden Krieges mit Deutschland in akute Lebensgefahr auch im benachbarten Frankreich brachte. „V.“, der Amateur-Biograph und Neu-Autor, der lächerliche Handelsvertreter, der sich als HobbyLiterat und -Literaturkritiker versucht, um Leben und Werke seines Halbbruders $\mathrm{zu}$ erklären, und damit letztlich scheitert, wird so zum axiologisch unzuverlässigen Erzähler. ${ }^{3}$ Sein biographischer Ästhetizismus kollidiert mit der Realität. So auch an einer anderen Stelle im Roman, zu Beginn des dritten Kapitels, als ,V.“ in einem Rückblick die Flucht der Halbbrüder und ihrer Mutter aus dem revolutionären Russland des Jahres 1918 schildert: Die Grenzen sind geschlossen, ihr Haus ist beschlagnahmt, der Schleuser, dem man das letzte Geld übergeben hat, wirkt gefährlich und wenig vertrauenserweckend. „V.“ schildert seine eigene Panik, als die Flucht bereits an ihrem Anfang zu scheitern droht. Da er Sebastians Gefühle nicht wiedergeben kann, montiert er eine Passage aus dessen (laut „V.“ autobiographischstem Werk) „Lost Property“ ein, in der Sebastian geschrieben hat: „A dark country, a hellish place, gentlemen, and if there is anything of which I am certain in life it is that I shall never exchange the liberty of my exile for the vile parody of home..." (Nabokov 1959 [1941], S. 27) Goodman, der Verfasser jener Biographie Sebastians, habe diese Passage als Zeitdokument gedeutet und einzig auf Russland bezogen, so „V.“ Gegen diese vereinnahmende Lesart wehrt sich ,V.“ jedoch vehement: ,[...] it should be quite clear to any unbiased reader that the quoted words refer rather to a fanciful amalgamation of tyrannic iniquities than to any particular nation or historical reality." (Nabokov 1959 [1941], S. 26) Warum einem als ,unvoreingenommenen Leser" diese Deutung so ,klar sein“ sollte, wird nicht ersichtlich. Es ist die Meinung der fiktiven Erzählerfigur „V.“, die zwar (wie auch Knight selbst) einige Züge des empirischen Autors Vladimir Nabokov trägt (u. a. den Anfangsbuchstaben seines Vornamens), andererseits aber als lächerliche, scheiternde Person erscheint und axiologisch unzuverlässiger Erzähler.

Nabokovs Roman ist also so konstruiert, dass letztlich auch Caultons Lesart, den Roman als Anschreiben gegen Totalitarismen aller Art und gegen die aus der Romantik überkommene Suche nach der ,absolute solution“ und nach dem ,verborgenen Geheimnis‘ des Lebens zu deuten, und die ästhetizistische Lösung, dieses Geheimnis einfach durch eine selbstbezügliche Kunst erst zu generieren, in wiederum typisch romantischer Ironie in der Schwebe bleibt.

\footnotetext{
${ }^{3}$ „Der fiktive Erzähler eines fiktionalen Erzähltextes ist genau dann axiologisch unzuverlässig, wenn seine Wertauffassungen den durch den Text im ganzen ausgedrückten Wertauffassungen nicht entsprechen“. (Köppe und Kindt 2014, S. 252 f.).
} 
Nabokovs Roman ist ein Kunstwerk, das seine Deutungsoffenheit in alle Richtungen und mit allen seinem Autor zu Gebote stehenden Mitteln absichert. Der ebenso mimetisch wie axiologisch nicht einmal offen unzuverlässige, sondern lediglich höchst zweifelhafte personale Ich-Erzähler ist hierfür nur das offenkundigste narrative Mittel.

\section{Grenzen des Verstehbaren. Thomas Manns Doktor Faustus}

Auch Thomas Manns Doktor Faustus. Das Leben des deutschen Tonsetzers Adrian Leverkühn, erzählt von einem Freunde ist ein „Roman des Exils“ (Vaget 2011, S. 443). Die Niederschrift erfolgte - eine dreizeilige erste Notiz aus der Zeit um 1904 aufgreifend - zwischen dem 23. Mai 1943 und dem 29. Januar 1947 (vgl. Wimmer 2007, S. 12 f., 23), mithin genau in der Zeit, in der auch der im Untertitel genannte „Freund[...]“ die Lebensgeschichte des Adrian Leverkühn abfasst (vgl. Ziegler 2015, S. 29). Mann wohnte in diesen Jahren im kalifornischen Pacific Palisades. Sein Aufenthaltsstatus war - im Gegensatz zu dem Nabokovs während der Arbeit am Sebastian Knight - relativ bald gesichert: 1944 wurde Mann, der seit 1933 in der Schweiz und seit 1938 dann in den USA gelebt hatte, die amerikanische Staatsbürgerschaft verliehen. Doktor Faustus erschien 1947 auf Deutsch im Exilverlag Bermann-Fischer in Stockholm. Mann hatte also den Sprachwechsel, der bei Nabokov zur Bedingung des Erfolgs seiner Auswanderungsbemühungen Richtung Amerika gewesen waren, selbst nie vollziehen müssen. 1949 kam dann mit Die Entstehung des Doktor Faustus. Roman eines Romans zudem ein Werk heraus, das als Manns Selbstkommentar die Arbeit am Roman beschreibt und das als Offenlegung autobiographischer Bezüge im Doktor Faustus, mithin als nicht-fiktionaler, autorpoetischer Text erscheint.

Der Roman ähnelt von seinem inhaltlichen Aufbau her zunächst dem von Nabokov: Ein Mann, der in seiner Jugend mit dem zum Zeitpunkt der Niederschrift bereits verstorbenen (fiktiven) Künstler privat verbunden war, macht sich daran, das Leben des Toten erzählend zu rekonstruieren, um sein Andenken für die Nachwelt zu bewahren und die verkannte Größe des Genies zu behaupten, für den toten Freund mithin einen Platz im kulturellen Gedächtnis zu beanspruchen. Wie bei Nabokov, so inszeniert sich auch bei Mann die fiktive Erzählerfigur als eine dem Künstler entgegengesetzte Natur - was wiederum die Frage aufwirft, inwieweit ein derart anders veranlagter Mensch überhaupt zu einem Verständnis des Portraitierten gelangen könne: ,hier der ehrgeizige, von faustischer Dämonie umwitterte und nach Suprematie strebende Künstler; dort der ganz und gar undämonische, exemplarisch bürgerliche Humanist und Chronist" (Vaget 2015, S. 70). „Metanarrative“ Passagen (vgl. Wißmach 2017, S. 173) aktualisieren immer wieder jenen auf die Romantik zurückgehenden existentiellen Gegensatz zwischen genialen, aber gefährdeten, auserwählten, inspirierten Künstlern auf der einen und künstlerisch gänzlich unbegabten, aber von Kunst und Kreativität 
amateurhaft angezogenen Alltagsexistenzen auf der anderen Seite. Man könnte sich fragen, warum sich dieser „Chronist“ überhaupt selbst derart ,in den Vordergrund“ der (fiktionalen) Biographie „schieb[t]“, wie er es z. B. als Eröffnung seiner Erzählung explizit tut:

Mit aller Bestimmtheit will ich versichern, daß es keineswegs aus dem Wunsche geschieht, meine Person in den Vordergrund zu schieben, wenn ich diesen Mitteilungen über das Leben des verewigten Adrian Leverkühn [...] einige Worte über mich selbst und meine Bewandtnisse vorausschicke. [...] nur weil ich damit rechne, daß man wünschen wird, über das Wer und Was des Schreibenden beiläufig unterrichtet zu sein, schicke ich diesen Eröffnungen einige wenige Notizen über mein eigenes Individuum voraus, - nicht ohne die Gewärtigung freilich, gerade dadurch dem Leser Zweifel zu erwecken, ob er sich auch in den richtigen Händen befindet, will sagen: ob ich meiner ganzen Existenz nach der rechte Mann für eine Aufgabe bin, zu der vielleicht mehr das Herz als irgendwelche berechtigende Wesensverwandtschaft mich zieht. (Mann 2007, S. 11; Hervorhebung von mir, S.K.)

Bereits zu Beginn seiner Erzählung legt Zeitblom jedoch offen, dass dieser Gegensatz seinem eigenen Wahrnehmungsmuster entspringt, nicht etwa dem des zur Gegenfigur aufgebauten Komponisten Leverkühn, dass mithin er selbst, nicht der Portraitierte, ein Romantiker ist:

Es war ein Künstlerleben; und weil mir, dem schlichten Manne, beschieden war, es aus solcher Nähe zu sehen, hat sich alles Gefühl meiner Seele für Menschenleben und -los auf diese Sonderform menschlichen Daseins versammelt. [...] Denn obwohl der Künstler seiner Kindheit zeitlebens näher [...] bleiben mag, als der im Praktisch-Wirklichen spezialisierte Mann; [...] so ist doch sein Weg [...] unendlich weiter, abenteuerlicher, für den Betrachter erschütternder, als der des bürgerlichen Menschen [...]. Dringend bitte ich übrigens den Leser, was ich da mit Gefühl gesagt habe, durchaus auf meine, des Schreibenden, Rechnung zu setzen und nicht etwa zu glauben, es sei in Leverkühns Sinne gesprochen. Ich bin ein altmodischer Mensch, stehen geblieben bei gewissen, mir lieben romantischen Anschauungen, zu denen auch der pathetisierende Gegensatz von Künstlertum und Bürgerlichkeit gehört. Adrian hätte einer Äußerung, wie der vorstehenden, kühl widersprochen [...]. (Mann 2007, S. 41 f.)

In der Forschung wurde diskutiert, ob der Roman letztlich ein „zweifache[s] Selbstporträt“" Thomas Manns mit dem „Janusgesicht seiner eigenen Existenz“ (Vaget 2015, S. 70) sei, wie man es auch in anderen Werken wie etwa Tonio Kröger gestaltet sehen könne: einerseits das einsame, gefährdete Genie, andererseits der etablierte Bildungsbürger. Beide Figuren, sowohl der kreative und experimentierende Künstler Adrian Leverkühn als auch der ein stilles Gelehrtenleben führende Humanist Serenus Zeitblom, tragen - das führt Mann in der Entstehung des Doktor Faustus deutlich vor - gewisse autobiographische Züge (vgl. Ziegler 2015, S. 21 f.). Doch beide sind auch eindeutig Kunstfiguren. Außerdem ist auch hier, wie in jedem Roman mit personal ausgestaltetem Erzähler, die ,Rede“ der Erzählerfigur zunächst einmal der einzige Zugang, den Leser zur fiktiven Welt des Romans und somit auch zu allen darin lebenden Figuren haben:

Alles, was der Leser des Doktor Faustus erfährt, erfährt er durch [...] den Biographen Serenus Zeitblom. Jede Interpretation muß zuallererst bei diesem Medium ansetzen, sich über seine perspektivisch ,brechenden" Eigenschaften orientieren und dabei den ungefähren Grad seiner Zuverlässigkeit bestimmen. (Klugkist 2000, S. 31) 
Der Roman ist denn auch von der Forschung der letzten 20 Jahre häufig vor allem aus narratologischer Perspektive betrachtet worden. Martínez und Scheffel (2012, S. 105) führen ihn in ihrer Einführung in die Erzähltheorie gar als paradigmatisches Beispiel für ,,[t]heoretisch unzuverlässiges“ Erzählen an. ${ }^{4}$ Kaiser (2001, S. 97) deutet ihn dagegen als ,mimetisch unzuverlässiges Erzählen“. Dabei kippt das mimetisch Offen-Unzuverlässige sogar manchmal ins TäuschendUnzuverlässige, etwa wenn Zeitblom vermerkt, dass er bei Adrians Bordellbesuch (Kap. XVI) sowie dem Teufelsgespräch (Kap. XXV) gar nicht dabei gewesen sei. Seine Versicherungen, sich für die detailreiche ,Ausmalung' dieser Szenen in seinem Bericht auf Briefe oder Manuskripte des Freundes zu berufen oder sich aufgrund seiner Kenntnis von Adrians typischen Verhaltensweisen eine bestimmte Situation sehr genau ,vor[zu]stellen“ zu können, wirken wie ein Bekenntnis zu der von Zweig praktizierten und theoretisierten Methode des biographischen ,Ausdeutens“ von vorhandenem Material (s. o.). Diese wirkt in Manns Roman jedoch nicht unproblematisch, wenn man bedenkt, dass die entsprechenden Passagen dann aber doch unmarkiert im Indikativ erzählt werden. ${ }^{6}$ Im weiteren Verlauf des Romans verliert Zeitbloms Selbstbescheidung als ,ausdeutender', nichts dazuerfindender Biograph dadurch immer mehr an Plausibilität. Immer häufiger werden mit dem Voranschreiten der erzählten Zeit diese Szenen im Leben des inzwischen erwachsenen Leverkühn, die Zeitblom - der Jugendfreund - nicht miterlebt hat und die er aber dennoch in allen Details zu erzählen weiß. Kaiser (2001, S. 98 f.) spricht von einer zunehmenden „Lizenz zum (allwissenden) Erzähler, die Zeitblom sich im Laufe des Romans selbst erteilt (bzw. die ihm sein Autor in wachsendem Maße zugesteht). " Häufiger kommt es dadurch

\footnotetext{
${ }^{4}$ Meine Hervorhebung, S.K. - Beim ,theoretisch unzuverlässigen Erzählen gibt die Erzählerfigur zwar die Fakten in der diegetischen Welt im Prinzip richtig wieder. Aufgrund ihres eigenen begrenzten Standpunktes in dieser Welt entgehen ihr aber (notwendigerweise) einige größere Zusammenhänge des erzählten Geschehens in der fiktiven Welt, z. B. „die politischen, philosophischen und moralischen Dimensionen ihrer eigenen Erzählung“ (Köppe und Kindt 2014, S. 252 f.).

${ }^{5}$ Beim ,mimetisch teilweise unzuverlässigen Erzählen“ können Leser nur dann eine konsistente Textwelt konstruieren, wenn sie einige der Aussagen über die fiktive Welt des Erzählers rückwirkend als falsch bewerten, um logische Widersprüche in der Erzählung aufzulösen (vgl. Martínez und Scheffel 2012, S. 105 f.).

${ }^{6}$ Aus narratologischer Perspektive liegt hier eine „Paralepse“ vor (vgl. Kaiser 2001, S. 96): So etwa beim Unterricht, bei dem mit „Adrian hielt ein Ohr hin, wandte sich ab und gab sich eine Miene, als wollte er sagen [...]“ genaue Details aufgezählt werden. Oder auch bei der zweiten, schicksalhaften Begegnung mit der Prostituierten ,hetaera esmeralda' (Kap. XIX), von der Zeitblom zwar zugibt, er sei nicht dabei gewesen, die er dann aber doch (sogar ,teilweise im Präsens“!) ganz genau beschreibt, was Zeitbloms „Rechtfertigung äußerst fadenscheinig wirken“ lässt (Kaiser 2001, S. 96), zumal, da der Erzähler hier sogar Innensicht in andere Personen simuliert: „Kein Zweifel, sie erinnerte sich des flüchtigen Besuchers von damals.“ (Alle Nachweise Kaiser 2001, S. 96) - Unter „Paralepse“ versteht man laut Genette (1994, S. 139) eine Erzählpassage, in der mehr Informationen gegeben werden, ,als der Fokalisierungscode, der das Ganze beherrscht, an sich gestattet.“
} 
auch zu den metanarrativen Kommentaren, die diese ,Allwissenheit‘ des Erzählers plausibilisieren sollen: „Zweifelt irgend jemand, daß ich, was zwischen Rudolf und Marie Godeau sich abspielte, in derselben Wörtlichkeit wiedergeben könnte, wie das Gespräch in Pfeiffering? Zweifelt jemand, daß ich ,dabei gewesen“ bin? Ich denke nicht.“ (Mann 2007, S. 641).

Der Diskurs der Erzählerfigur Zeitblom kann aber auch als mimetisch ,uneindeutiges Erzählen“ bezeichnet werden: Immer wieder macht Zeitblom ja offen darauf aufmerksam, dass er als einfacher Mann die Gedankenwelt des genialen Künstlers möglicherweise nur unzureichend und durch die ,falsche Brille ' hindurch betrachten und daher auch eventuell nur verzerrt wiedergeben könne. ${ }^{7}$

Thomas Mann selbst hat sich in dem ganzen Konglomerat von „essayistischen Begleittexten“ (Vaget 2015, S. 71), die sich mit der ,Entstehung des Doktor Faustus ' beschäftigen, immer wieder zu dieser homodiegetischen Erzählerfigur des Serenus Zeitblom geäußert. Offenbar soll dieser Erzähler nicht zuverlässig und allwissend erscheinen, sondern eine komische, für den Leser in seiner Unzuverlässigkeit durchschaubare Figur darstellen (so zumindest im Tagebuch unter dem 02.06.1943). ${ }^{8}$ Zudem solle die zusätzliche Zeitebene, die durch Zeitbloms Erfahrungen der Bombenangriffe $\mathrm{zu}$ dem Romangeschehen hinzutritt, die Deutschland-Thematik ${ }^{9}$ verstärken und sich, so Mann wörtlich, ,polyphon“ mit den früheren Zeitebenen ,,verschränken“, ${ }^{10}$ aber eben auch mit der außertextuellen Gegenwart des empirischen Autors. Das Kapitel „XLIII“ - eines der letzten des Romans - beginnt Zeitblom mit den Worten:

\begin{abstract}
Meine Erzählung eilt ihrem Ende zu - das tut alles. Alles drängt und stürzt dem Ende entgegen, in Endes Zeichen steht die Welt, - steht darin wenigstens für uns Deutsche, deren tausendjährige Geschichte, widerlegt, ad absurdum geführt, als unselig verfehlt, als Irrweg erwiesen durch dieses Ergebnis, ins Nichts, in die Verzweiflung, in einen Bankerott ohne Beispiel, in eine von donnernden Flammen umtanzte Höllenfahrt mündet. [...] Ich, ein schlichter deutscher Mann und Gelehrter, habe viel Deutsches geliebt, ja, mein unbedeutendes, aber der Faszination und Hingabe fähiges Leben war der Liebe, der oft verschreckten, der immer bangen, aber in Ewigkeit getreuen Liebe zu einem bedeutend deutschen Menschen- und Künstlertum geweiht, dessen geheimnisvolle Sündhaftigkeit
\end{abstract}

\footnotetext{
${ }^{7}$ Vgl. die Ausführungen von Tom Kindt im vorliegenden Band.

${ }^{8}$ Zitiert nach Kaiser (2001, S. 92).

${ }^{9}$ Bereits im Frühjahr 1943 bat der Verleger Bermann Fischer Mann um ,ein Buch über Deutschland, Vergangenheit und Zukunft“ (zitiert nach Wimmer 2007, S. 15). Mann verwebt diese Idee umgehend mit dem Faust-Stoff, mit dem er sich in dieser Zeit bereits beschäftigt hat, und der alten Projekt-Skizze für eine Geschichte über einen „syphilitischen Künstler“ (Wimmer 2007, S. 13).

${ }^{10}$ Zitiert nach Kaiser (2001, S. 93). Der Roman behandele somit die „prä-faschistische Zeitspanne von 1885-1930“ sowie die Zeit „,des Niedergangs des deutschen Nationalsozialismus“ von „1943-1945“. (Kaiser 2001, S. 93) - Ob diese von Kaiser auf das Politische festgelegte Deutung trägt, wäre zu prüfen. Ziegler (2015, S. 29) macht immerhin darauf aufmerksam, dass Adrians Schaffenszeit (1906-1930) auch die Zeit der europäischen Avantgarden, der ästhetisch experimentellen Kunst ist.
} 
und schrecklicher Abschied nichts über diese Liebe vermögen, welche vielleicht, wer weiß, nur ein Abglanz der Gnade ist. (Mann 2007, S. 654 f.)

Die fiktive Erzählerfigur Zeitblom - nicht Thomas Mann selbst - deutet Adrians Schicksal als ,pars pro toto“: Der „deutsche[...] Tonsetzer“ (meine Hervorhebung, S.K.), der den Pakt mit dem Teufel eingegangen sei, den auch die Deutschen 1933 mit dem ,Teufel` Hitler geschlossen hätten, wird für Zeitblom zur Allegorie ${ }^{11}$ für das „Heraufsteigen und Umsichgreifen dessen [...], das sich dann des Landes bemächtigte und nun [1945] in Blut und Flammen untergeht" (Mann 2007, S. 698 f.) zu einem Zeitpunkt, da Adrian längst tot ist. Aber ist das auch die Werkbedeutung? Zusammengenommen sind Doktor Faustus und das Buch über seine Entstehung als Manns Abrechnung mit der einst „,von ihm selbst verherrlichten deutschen Musikkultur“ gedeutet worden, aus der der „kulturell begründete Superioritätswahn" hervorgegangen sei, der dem schließlich so fatalen „deutschen Weltmachtstreben“ zugrunde gelegen habe (so z. B. von Vaget 2011, S. 446). Crescenzi (2017) geht sogar so weit, Die Entstehung des Doktor Faustus als Manns persönliche, nicht-fiktionale, in eigenem Namen getätigte Abrechnung mit seiner früheren, ironisch-ästhetizistisch-unpolitischen Kunst und mit dem autofiktionalen Rollenspiel in seinen literarischen Werken (einschließlich des Doktor Faustus') zu sehen. Doch gegen diese Interpretation ist einzuwenden, dass die Referenzbehauptung der in der Entstehung getätigten Aussagen letztlich auch wieder in der Schwebe gehalten werden: Durch den im typisch romantischen Genitiv stehenden Untertitel „Roman eines Romans“ wird auch der scheinbar autorfaktuale Bericht über die Arbeit an der fiktionalen Biographie des fiktiven Künstlers Adrian Leverkühn wieder fiktionalisiert - und das, obwohl es sich um einen Text handelt, der im Ganzen nur aus Propositionen des empirischen Autors Thomas Mann zu bestehen scheint, der sich auf empirische Tagebücher stützt und der laut Selbstaussage seines Verfassers dem gänzlich nicht-fiktionalen Zweck dienen sollte, die musikalische Mitarbeit Theodor W. Adornos offenzulegen (vgl. Schoenberg 2009, S. 101) ${ }^{12}$ und den Verfasser somit vom Vorwurf des Plagiats reinzuwaschen (vgl. Wimmer 2007, S. 9 f.). Ratlos standen zahlreiche Rezensenten denn auch vor diesem fiktionalen Werk und seinem ,Making-of': Ist das nun ein politisches Statement im Angesicht der ,deutschen Katastrophe " von 1933 bis 1945 oder herrscht hier erneut nur „ein heilloser Relativismus“?"13 (Vaget 2015, S. 72).

\footnotetext{
${ }^{11}$ Wäre der Text phantastisch, so wäre es sein zentrales „Thema“, dass seine Ontologie (gibt es den Teufel in der fiktiven Welt des Romans oder nicht?) fraglich bliebe (vgl. Kindt 2011, S. 53, 55). Schon Todorov grenzte die Allegorie jedoch klar von der Phantastik ab: Bei einer Allegorie geht es nicht primär um die Frage nach der Ontologie einer fiktiven Welt, sondern um Aussagen über die reale Welt (vgl. Todorov 2013, S. 70-80).

${ }^{12}$ Und ein bisschen wohl auch, um dem Urheberrechtsstreit mit Arnold Schönberg über die Erfindung der im Roman verarbeiteten Zwölftonmusik zu entgehen (vgl. Todorov 2013, S. 106113).
}

${ }^{13}$ Ein knapper Überblick über die zeitgenössische Rezeption findet sich bei Vaget (2015, S. 72). 
Der Zweifel, die universale Ironie, die im Roman über der ,komischen“ Figur Zeitblom hängt und die der „Roman eines Romans“ nicht auszuräumen vermag, lässt also auch die axiologische Zuverlässigkeit der Beschreibung Zeitbloms von Leverkühns letzter Komposition, der „Symphonischen Kantate ,Dr. Fausti Weheklag “ (Mann 2007, S. 699), in der Schwebe bleiben:

\begin{abstract}
Nein, dies dunkle Tongedicht läßt bis zuletzt keine Vertröstung, Versöhnung, Verklärung zu. Aber wie, wenn der künstlerischen Paradoxie, daß aus der totalen Konstruktion sich der Ausdruck - der Ausdruck der Klage - gebiert, das religiöse Paradoxon entspräche, daß aus tiefster Heillosigkeit, wenn auch als leiseste Frage nur, die Hoffnung keimte? Es wäre die Hoffnung jenseits der Hoffnungslosigkeit, die Transzendenz der Verzweiflung, - nicht der Verrat an ihr, sondern das Wunder, das über den Glauben geht. Hört nur den Schluß, hört ihn mit mir: Eine Instrumentengruppe nach der anderen tritt zurück, und was übrigbleibt, womit das Werk verklingt, ist das hohe g eines Cello, das letzte Wort, der letzte verschwebende Laut, in pianissimo-Fermate langsam vergehend. Dann ist nichts mehr. Schweigen und Nacht. Aber der nachschwingend im Schweigen hängende Ton, der nicht mehr ist, dem nur die Seele noch nachlauscht, und der Ausklang der Trauer war, ist es nicht mehr, wandelt den Sinn, steht als ein Licht in der Nacht. (Mann 2007, S. 711)
\end{abstract}

„Licht“ für Deutschland und die Deutschen, 1945? Eingriff göttlicher Gnade, weil der Teufelsbündler so arg weheklagt? Also doch das Ende aus Goethes Faust II, Rettung und Himmelfahrt statt Höllenfahrt und ewiger Verdammnis, wie im mittelalterliche Volksbuch? Adorno, Thomas Manns engster Berater in musikwissenschaftlichen Fragen, zeigte sich bekanntlich schockiert; doch ganz unplausibel ist diese Deutung nicht, nimmt das Thema der „Gnade selbst für den extremsten Sünder" doch in Manns letzten Schriften tatsächlich einen immer größeren Raum ein (vgl. Vaget 2015, S. 74).

An diesem heiklen, in der Rezeptionsgeschichte des Romans bis heute umstrittenen Punkt merkt man, welche Funktion die Erzählkonstruktion mit der Erzähler-Figur des Zeitblom neben allem anderen eben auch zukommt: In Zeitbloms offener, teilweise mimetischer, eventuell auch axiologischer Unzuverlässigkeit schafft sich Thomas Mann die Möglichkeit, unter dem Vorzeichen der Fiktion eine These auszusprechen, eine Idee anklingen zu lassen, ohne dadurch doch eine vorschnelle, billige Hoffnung zu propagieren. Es ist jedoch auch ein weiterer Beleg für die künstlerische Haltung des Ästhetizismus im Werk Thomas Manns, auch nach 1945 noch. Unter dem Deckmantel dieser ,komischen“ Figur Zeitblom kann Thomas Mann 1947, als im amerikanischen Exil längst Eingebürgerter Ex-Deutscher unter Kommunismus-Verdacht, Aussagen tätigen, die er als Propositionen wohl nie in eigenem Namen getätigt hätte, die aber unter dem Vorzeichen der Fiktion und relativiert durch die offene Unzuverlässigkeit der Erzählerfigur gleichwohl in den Raum gestellt und somit diskutierbar gemacht werden.

\title{
3 Fazit
}

Thomas Manns Doktor Faustus und Vladimir Nabokovs Real Life of Sebastian Knight sind zwei Klassiker des mimetisch wie axiologisch unzuverlässigen oder zumindest fragwürdigen Erzählens. Beide rekurrieren auf Formen des literarischen 
,Life-Writing“: Beide sind fiktionale Romanbiographien über fiktive Künstler, die aus der Perspektive des Nichtkünstlers den romantischen Gegensatz zwischen Genie und Normalbürger ebenso in Zweifel ziehen wie die Suche nach einer alle Rätsel auflösenden Weltformel. Beide Romane sind hochgradig metafiktional und selbstreferentiell, reflektieren permanent das eigene ästhetizistische Erbe (ohne sich doch je entschieden davon zu distanzieren) und verweben erkennbar autobiographische Realien in verschiedenen, einander eigentlich entgegengesetzten Figuren. Inhalt und narrative Struktur der Romane sind also durchaus vergleichbar.

Thomas Mann und Vladimir Nabokov sind sich vermutlich nie begegnet. $\mathrm{Ob}$ Mann von Nabokov Notiz nahm, ist nicht bekannt. Als Ende der 1950er Jahre die Bekanntheit des Exil-Russen durch den Skandalroman Lolita auch in Europa stieg, war Mann bereits verstorben. Von Nabokov dagegen sind heftige Invektiven gegen Manns literarischen Werke überliefert (vgl. z. B. Nabokov 1965/2017, S. 96). ${ }^{14}$ Von einem positivistisch nachweisbaren Einfluss des älteren Werks auf das jüngere kann also vermutlich keine Rede sein. Vergleichbare literarische Werke werden hier aber unter in Ansätzen vergleichbaren biographischen Situationen geschaffen: Das Spiel mit den Konventionen der in den 1920er und 1930er Jahren so populären, anti-modernen Gattung der Romanbiographie bezieht auch die Lesererwartung, einen Schlüsselroman oder aber ästhetizistische ,absolute Kunst ${ }^{\text {‘ }}$ in Händen zu halten, mit ein. Die Romane reflektieren jeder auf seine Weise die Bedingungen der Möglichkeit des Schreibens von Biographien, die Grundaporie des ,Life-Writings“ überhaupt: Wie kann man ein Leben oder Werk verstehen? Gibt es einen verborgenen Sinn hinter den ,Zeichen“ und Zufällen eines Menschenlebens? Ist ein Leben überhaupt erzählbar (d. h. außer in Ereignisse temporaler Ordnung auch mit einem größeren narrativen Bogen zu versehen, einer sinnhaften Verknüpfung zwischen einzelnen Szenen und Episoden und schließlich auch mit einem sinnhaften Ende, einer ,narrative closure ${ }^{6} \mathrm{zu}$ versehen)? ${ }^{15}$ Und wie wäre eine solche Lebenserzählung überzeugend zu vermitteln, wer könnte sie überhaupt realistischerweise erzählen?

Die beiden Romane verfügen über die literarische Tradition der Romantik wie der Moderne, sie problematisieren die menschliche Sehnsucht nach der , absoluten Lösung' und fallen letztlich doch auf die ästhetizistische Variante, diese Lösung einfach durch Kunst zu generieren, zurück. Beide Werke lassen sich - trotz der

\footnotetext{
${ }^{14}$ Bei Nabokov stellt sich zudem immer die Frage: Wie gut kannte er die deutsche Literatur? Lolita ist beispielsweise als die Aus- oder Umarbeitung einer Novelle verstanden worden, die ein gewisser Heinz von Lichberg 1916 auf Deutsch veröffentlicht hatte und in der es auch um ein minderjähriges Mädchen namens Lolita geht, durch das sich der ältere männliche IchErzähler zu sexuellen Handlungen angeregt fühlt (vgl. Krause 2004). - Maar (2007) hat in einer vergleichenden Studie von Thomas Manns Erzählung Der kleine Herr Friedemann (1897) mit Nabokovs Der Kartoffelelf (1924) gezeigt, dass Nabokov durchaus „,genaue Kenntnis“ des Mann'schen Oeuvres besessen habe und dass er in dem für seine Sprachartistik gerühmten Mann eine „Konkurrenz“ gesehen habe, die es zu ,überbieten“ gegolten habe.

${ }^{15} \mathrm{Vgl}$. zu einem diese Aspekte enthaltenden Verständnis von ,Erzählung ' Köppe und Kindt (2014, S. 64-70).
} 
dringlichen politischen Lage, in der sie entstanden - nicht auf eine klare Werkbedeutung festlegen. Nicht einmal mithilfe der letztlich selbst wieder durch den Untertitel fiktionalisierten ,Selbstdeutung' Manns in der Entstehung des Doktor Faustus. Nabokov, auf dem Weg in ein neues Exil, zeigt seine Kunst, ohne sich als Vertreter einer bestimmten Meinung zu exponieren. Mann, eingebürgert und dennoch beargwöhnt in den USA, die er schon bald wieder für einen Lebensabend in seinem letzten ,Exil', der Schweiz, verlassen wird, ${ }^{16}$ macht - ironisch verklausuliert - einen Meinungsvorschlag, ohne sich selbst als Person dazu zu bekennen. Gab es für die einmal von der unendlichen Selbstbespiegelung der Kunst erfassten Autoren kein Entkommen mehr zurück in die Realität? Oder handelt es sich bei diesen beiden Romanen womöglich schlicht um zwei weitere Belege für die von J.M. Coetzee vorgebrachte These, der Exilant sei eben stets ein strenger Selbstzensor (vgl. Ziegler 2015)? - Der Interpret wird beim Versuch, diese Fragen zu beantworten, vermutlich auch wieder einmal mehr über sich selbst als über die Romane aussagen.

\section{Literatur}

Boyd, Brian: Vladimir Nabokov. Vol. 1: The Russian Years. Princeton, New Jersey: Princeton UP, 1990.

Caulton, Andrew: The Absolute Solution. Nabokov's Response to Tyranny, 1938. Oxford u. a.: Lang, 2013.

Crescenzi, Luca: Masken. Zu den Strategien der Selbstbiografik im Doktor Faustus und in der Entstehung des Doktor Faustus. In: Thomas Mann Jahrbuch 30 (2017), 87-98.

Genette, Gérard: Die Erzählung. Aus dem Französischen von Andreas Knop, mit einem Nachwort hrsg. v. Jochen Vogt. München: Fink, 1994.

Fricke, Harald: Potenzierung. In: Reallexikon der deutschen Literaturwissenschaft. Bd. III, JanDirk Müller (Hg.). Boston u. Berlin: de Gruyter, 2002, 144-147.

Kaiser, Gerhard: „... und sogar eine alberne Ordnung ist immer noch besser als gar keine. “ Erzählstrategien in Thomas Manns, Doktor Faustus'. Stuttgart, Weimar: Metzler, 2001.

Kindt, Tom: „Das Unmögliche, das dennoch geschieht“. Zum Begriff der literarischen Phantastik am Beispiel vom Werken Thomas Manns. In: Thomas Mann Jahrbuch 24 (2011), 43-56.

King, Martina: Vom ,heiligen Schwips'. Medizinisches Wissen und kunstreligiöse Tradition in den Inspirationsszenarien von Der Zauberberg und Doktor Faustus. In: Thomas Sprecher (Hg.): Zwischen Himmel und Hölle. Thomas Mann und die Religion. Frankfurt a. M.: Klostermann, 2012, 53-83.

Klugkist, Thomas: Sehnsuchtskosmogonie. Thomas Manns Doktor Faustus im Umkreis seiner Schopenhauer-, Nietzsche- und Wagner-Rezeption. Würzburg: Königshausen \& Neumann, 2000.

Köppe, Tilmann, u. Tom Kindt: Erzähltheorie. Eine Einführung. Stuttgart: Reclam, 2014.

\footnotetext{
${ }^{16}$ Abgestoßen durch das zunehmend feindselige Klima des beginnenden Kalten Krieges zog Thomas Mann 1952 mit einem Teil seiner Familie in den Kanton Zürich und starb 1955 in seiner Villa in Kilchberg am Zürichsee. Vladimir Nabokov zog 1961 ebenfalls in die Schweiz und verbrachte seine letzten 16 Lebensjahre gemeinsam mit seiner Frau Vera als Dauermieter im Grandhôtel Palace in Montreux am Genfersee.
} 
Krause, Tilman: Haben Nabokov und Thomas Mann geklaut? In: Die Welt 04.05.2004. URL: https://www.welt.de/print-welt/article311124/Haben-Nabokov-und-Thomas-Mann-geklaut. html (22. Dezember 2018).

Lee, Hermione: The Novels of Virginia Woolf [1977]. Abington: Routledge Revivals, 2010.

Maar, Michael: Solus Rex. Die schöne böse Welt des Vladimir Nabokov. Berlin: Berlin Verlag, 2007.

Mann, Thomas: Doktor Faustus. Das Leben des deutschen Tonsetzers Adrian Leverkühn, erzählt von einem Freunde. Bd. 10.1 der Großen kommentierten Frankfurter Ausgabe Thomas Manns. Werke - Briefe - Tagebücher. Heinrich Detering u. a. (Hg.). Frankfurt a. M.: Fischer, 2007.

Martínez, Matías, u. Michael Scheffel: Einführung in die Erzähltheorie. 9., erw. u. aktual. Aufl. München: Beck, 2012.

Nabokov, Vladimir: Das wahre Leben des Sebastian Knight. Roman. Deutsch von Dieter E. Zimmer. Neuausgabe. Reinbek bei Hamburg: Rowohlt, 1999.

Nabokov, Vladimir: The Real Life of Sebastian Knight. New York: New Directions, 1959 [1941].

Nabokov, Vladimir: Conversations with Vladimir Nabokov. Robert Golla (Hg.). Jackson: UP of Mississippi, 2017.

Schoenberg, E. Randol (Hg.): Apropos Doktor Faustus. Briefwechsel Arnold Schönberg Thomas Mann, Tagebücher und Aufsätze 1930-1951. Übersetzungen von Susanne Müller und Elisabeth Schwagerle. Wien: Szernin, 2009.

Todorov, Tzvetan: Einführung in die fantastische Literatur. Übersetzt aus dem Französischen von Karin Kersten, Senta Metz und Caroline Neubaur. Berlin: Wagenbach, 2013.

Vaget, Hans Rudolf: Doktor Faustus. In: Thomas Mann Handbuch. Leben - Werk - Wirkung. Hg. Andreas Blödorn und Friedhelm Marx. Stuttgart: Metzler, 2015, 66-75.

Vaget, Hans Rudolf: Thomas Mann, der Amerikaner. Leben und Werk im amerikanischen Exil 1938-1952. Frankfurt a. M.: Fischer, 2011.

Wimmer, Ruprecht, unter Mitarbeit von Stephan Stachorski: Kommentar zu Thomas Mann: Doktor Faustus. Das Leben des deutschen Tonsetzers Adrian Leverkühn, erzählt von einem Freunde. Bd. 10.2 der Großen kommentierten Frankfurter Ausgabe Thomas Manns. Werke Briefe - Tagebücher. Hrsg. v. Heinrich Detering u. a. Frankfurt a. M.: Fischer, 2007.

Wißmach, Friederike: „Erwägen wir die Möglichkeiten“. Zum Problem der narrativen Sinnstiftung in den Josephs-Romanen und im Doktor Faustus. In: Regine Zeller, Jens Ewen u. Tim Lörke (Hg.): Der Geist der Erzählung. Narratologische Studien zu Thomas Mann. Würzburg: Königshausen \& Neumann, 2017, 169-193.

Ziegler, Heide: Exile and Self-Censorship: Thomas Mann and Vladimir Nabokov. In: Censorship and Exile. Hg. Johanna Hartmann u. Hubert Zapf. Göttingen: V\&R Unipress, 2015, 13-36.

Zimmer, Dieter E.: Bierkrüge und Vergissmeinnicht Nabokovs Abneigung gegen Deutschland und die Deutschen. Vortrag, gehalten am 23.10.1999 im Nabokov-Museum, St. Petersburg. URL: https://www.d-e-zimmer.de/PDF/nabokov+deutschland1999.pdf (22. Dezember 2018).

Zimmer, Dieter E.: Nachwort des Herausgebers. In: Vladimir Nabokov: Das wahre Leben des Sebastian Knight. Roman. Deutsch von Dieter E. Zimmer. Reinbek bei Hamburg: Rowohlt, 1999 [1960], 265-299.

Zweig, Stefan: Die Geschichte als Dichterin. In: Bernhard Fetz u. Wilhelm Hemecker (Hg.): Theorie der Biographie. Grundlagentexte und Kommentar. Berlin u. New York: de Gruyter, 2011. 
Open Access Dieses Kapitel wird unter der Creative Commons Namensnennung 4.0 International Lizenz (http://creativecommons.org/licenses/by/4.0/deed.de) veröffentlicht, welche die Nutzung, Vervielfältigung, Bearbeitung, Verbreitung und Wiedergabe in jeglichem Medium und Format erlaubt, sofern Sie den/die ursprünglichen Autor(en) und die Quelle ordnungsgemäß nennen, einen Link zur Creative Commons Lizenz beifügen und angeben, ob Änderungen vorgenommen wurden.

Die in diesem Kapitel enthaltenen Bilder und sonstiges Drittmaterial unterliegen ebenfalls der genannten Creative Commons Lizenz, sofern sich aus der Abbildungslegende nichts anderes ergibt. Sofern das betreffende Material nicht unter der genannten Creative Commons Lizenz steht und die betreffende Handlung nicht nach gesetzlichen Vorschriften erlaubt ist, ist für die oben aufgeführten Weiterverwendungen des Materials die Einwilligung des jeweiligen Rechteinhabers einzuholen. 